\title{
BMJ Open Characterising fetal alcohol spectrum disorder in Canada: a national database protocol study
}

\author{
Jocelynn Cook (D) , ${ }^{1}$ Kathy Unsworth, ${ }^{2}$ Katherine Flannigan (iD ${ }^{3}$
}

To cite: Cook J, Unsworth K, Flannigan K. Characterising fetal alcohol spectrum disorder in Canada: a national database protocol study. BMJ Open 2021;11:e046071. doi:10.1136/ bmjopen-2020-046071

- Prepublication history and additional supplemental material for this paper are available online. To view these files, please visit the journal online (http://dx.doi.org/10.1136/ bmjopen-2020-046071).

Received 25 November 2020 Accepted 17 August 2021

Check for updates

(c) Author(s) (or their employer(s)) 2021. Re-use permitted under CC BY-NC. No commercial re-use. See rights and permissions. Published by BMJ.

${ }^{1}$ Society of Obstetricians and Gynaecologists of Canada, Ottawa, Ontario, Canada

${ }^{2}$ The Canada FASD Research Network, Ottawa, Ontario, Canada

${ }^{3}$ The Canada FASD Research Network, Edmonton, Alberta, Canada

Correspondence to

Dr Jocelynn Cook;

jcook@sogc.com

\section{ABSTRACT}

Introduction Fetal alcohol spectrum disorder (FASD) is one of the most common neurodevelopmental disorders in North America. It is a complex disability, associated with challenges in cognitive, behavioural and socialemotional functioning, as well as an increased risk of physical and mental health comorbidities, and difficulties in daily living across the lifespan. Previous attempts to characterise the profile of this population have been hampered by differences in data collected across studies, regional discrepancies in terminology and definitions, and a lack of tools to integrate comprehensive datasets.

Methods and analysis The goals of this study are to use the Canadian National FASD Database, a national repository of FASD assessment-related information, to better understand the functional profile, comorbidities, intervention needs and difficulties in daily living experienced by individuals assessed for FASD across the lifespan. We will also examine what factors may be the most sensitive predictors of receiving an FASD diagnosis. Data will be analysed from over 3500 records collected between 2010 and 2021 (ongoing) from 26 FASD diagnostic clinics in seven provinces and territories. Data collection is ongoing, and analysis will be performed on a biannual basis to continue to hone our understanding of the profiles, needs and outcomes of individuals assessed for FASD in Canada. This research is critical for refining FASD assessment and diagnostic practice, enabling accurate and early identification of individuals with FASD, and connecting individuals with FASD and their families to comprehensive and effective services and resources to support healthy developmental trajectories.

Ethics and dissemination Ethics approval for the National FASD Database Project was obtained from the Ottawa Health Science Network Research Ethics Board. As new knowledge is gained from this project, findings will be disseminated through publications, presentations and feedback to participating clinics, with the ultimate goal of informing FASD research, practice and policy.

\section{INTRODUCTION}

\section{Health and human development}

Health vulnerability and associated developmental trajectories are rooted in the prenatal stage and first years of life, both of which are critical periods involving complex interactions between biological, genetic and environmental conditions. Many determinants

\section{Strengths and limitations of this study}

- The Canadian National Fetal Alcohol Spectrum Disorder (FASD) database is the first and only existing standardised patient-level database of individuals assessed for FASD in Canada, which allows for the identification of trends related to the prevalence and diagnosis of FASD and associated features.

- Ongoing data collection enables the monitoring of changes in population-level profiles, needs and experiences of individuals assessed for FASD in Canada, as well as access to timely information to guide FASD research, practice and policy.

- The database was developed in consultation with government stakeholders, clinicians, researchers and individuals with FASD and their families, ensuring that information collected is relevant and meaningful for individuals with FASD and those who support them.

- Data are collected from many, but not all, clinics in Canada, and there are several provincial and territorial jurisdictions that are not represented in the database.

- Information collected is cross-sectional, limiting our ability to explore longitudinal trends or follow the developmental trajectories of individuals with FASD across the lifespan.

of health contribute to optimal development and are relevant for all human beings, regardless of culture or background. Maternal and fetal health, the early caregiving environment and family influences, poverty and malnutrition, neighbourhood factors and the broader sociopolitical context can all have profound impacts on human development and healthy outcomes. ${ }^{1}$ In the long term, poor physical, mental and socioemotional development in childhood is linked to unfavourable outcomes such as school failure, delinquency, unemployment and poor health in adulthood. ${ }^{2}$

Researchers have worked hard to identify permissive and protective factors that optimise developmental outcomes, from preconception through to adulthood. The presence of a diagnosable medical condition early in 
life can greatly impact an individual's health trajectory throughout the lifespan. ${ }^{3}$ Data strongly suggest that providing early interventions and supports can have protective effects, mitigate difficulties in daily living and provide a foundation for healthier trajectories. ${ }^{4}$ However, in order to achieve these benefits, it is essential that individuals who are at risk of negative outcomes are accurately identified and connected with appropriate and effective supports.

\section{Developmental trajectories and prenatal alcohol exposure}

Prenatal alcohol exposure (PAE) is associated with a broad range of neurodevelopmental and behavioural needs which, without standardised mechanisms for identification, can remain unaddressed. When needs are not recognised, individuals with PAE can experience substantial challenges, and critical opportunities for early interventions to improve outcomes for individuals and families may be missed. ${ }^{5}$ Indeed, researchers have shown that early identification and intervention are some of the most powerful factors to mitigate the lifelong adverse effects of PAE. ${ }^{46}$

Because of the complex and heterogeneous consequences of PAE, a standardised data collection protocol using common data fields can be a powerful and comprehensive tool for understanding PAE and its associated impacts. At a national level, such a protocol allows for the large-scale examination of the neurodevelopmental effects of PAE, as well as the identification of other social and environmental factors that may influence outcomes for individuals with PAE. Moreover, it can improve our understanding of the supports, strategies and interventions that may reduce challenges and optimise growth and potential for positive outcomes for individuals with PAE and their families.

\section{Fetal alcohol spectrum disorder}

When the brain-based and body-based impacts of PAE reach a clinical threshold, individuals may be diagnosed with fetal alcohol spectrum disorder (FASD). ${ }^{7}$ FASD is a lifelong disability associated with difficulties in motor function, learning, memory, attention, communication, emotional regulation and social skills. Individuals with FASD often require ongoing support with daily living and are at high risk for compromised developmental trajectories, stemming from the neurodevelopmental impacts of PAE, compounded by complex biopsychosocial and societal factors and societal. Individuals with FASD often have extensive patterns of impairment with co-occurring physical and mental health conditions that influence their clinical presentation, treatment recommendations and potential outcomes. ${ }^{5}$ 8-10 They also often experience early ${ }^{11}$ and ongoing environmental adversity ${ }^{5} 612$ and disruption in the caregiving environment ${ }^{13} 14$ which can impact social, behavioural and emotional development. ${ }^{13} 1516$ Difficulties with daily living are common among individuals with FASD, including problems with school and employment; independence and housing; mental health and substance use challenges; and interaction with the justice system. ${ }^{56}$ That said, there is very limited research that focuses on the strengths of individuals with FASD, and how to achieve successful outcomes, and there is a critical need to develop and implement strengths-based approaches and interventions for this population. ${ }^{17}$ For example, in one study, researchers identified predictive factors that contribute to success in occupational performance in youth and adults with FASD, ${ }^{18}$ and in another small study reported on factors that influence success in school, ${ }^{19}$ and others have reported on factors that contribute to positive outcomes among adults with FASD who are involved in the justice system. ${ }^{20}$

FASD affects approximately $4 \%$ of the Canadian population and is a complex social and public health issue..122 Individuals with FASD are an exceptionally complex and heterogeneous group, and there is a strong interest among researchers and clinicians in characterising the profiles, needs and experiences of these individuals. ${ }^{23} 24$ However, there are challenges with characterising individuals with FASD, such as inconsistent definitions of the disability, varying diagnostic systems and approaches, as well as the resource-intensive multidisciplinary diagnostic process itself. Attempts to compare data across FASD studies have largely failed because of the discrepancies in these definitions and approaches. These challenges highlight the potential utility of a consistent, nationwide database to inform FASD research, practice and policy.

\section{Measuring FASD at the population level in Canada}

In Canada, there is a paucity of population-level information about individuals with PAE and FASD, which is critical for building meaningful, cost-effective and appropriately distributed programming and interventions. Over the past decade, Canadian researchers have sought to address this gap by working together to develop and contribute to a standardised database with a common set of indicators. The Universal FASDataForm Project was initiated in 2010 in collaboration with Canadian FASD diagnostic clinics to determine if standardised collection of assessmentrelated data was a possibility, and then subsequently to generate the first clinical dataset for FASD, and identify trends and modalities related to prevention, prevalence and diagnosis of FASD. ${ }^{25}$ The FASDataForm was revised in 2015 to refine the process of collecting and comparing common data indicators, resulting in the updated (and renamed) National FASD Database Project. The main purpose of the Database Project is to capture information related to the assessment and diagnosis of FASD in Canada, including information on the physical and mental health needs, functional challenges and difficulties in daily living experienced by individuals presenting for FASD assessment across the country.

In the current study, our goal is to investigate the profile and experiences of individuals assessed for FASD in Canada. Analysis of data from the database will allow us to interpret and disseminate findings on characteristics, associated features and experiences of individuals 
presenting for an FASD assessment. The study is guided by the following research questions:

- What is the neurodevelopmental profile of individuals assessed for FASD in Canada? How does it compare to profiles of individuals assessed for FASD in other countries?

- What are the physical and mental health comorbidities associated with FASD? How do these rates compare to the general population?

- What are the most sensitive predictive factors for an FASD diagnosis?

- Which non-diagnostic factors are the most strongly predictive of FASD?

- Which diagnostic and individual factors are the most strongly predictive of FASD?

- What are the most common recommendations for interventions for individuals assessed for FASD?

- What factors may contribute to or protect against the difficulties in daily living associated with FASD?

\section{METHODS AND ANALYSIS}

\section{Data source and variables}

The National FASD database is an ongoing data repository composed of clinical and diagnostic findings for individuals of all ages presenting for an FASD assessment to participating clinics $(n=26)$ from seven provinces and territories in Canada. The database contains responses from a 287-item bilingual (English or French) questionnaire, completed online through the REDCap platform, usually by a clinic intake coordinator. Data fields are populated based on chart review of each individual who has completed the FASD assessment process. The database includes records generated over two data collection periods between 2010 and 2021, with ongoing entry.

The database captures a wide range of information including individual demographics, referral source and reasons for referral, living situation, family history of FASD, prenatal exposure to alcohol and other teratogens, and early life adversity. Aligning with the current Canadian Diagnostic Guideline criteria, ${ }^{7}$ data are recorded for each individual on confirmation of PAE above risk levels (Under the Canadian Diagnostic Guideline, aboverisk PAE threshold is defined as $\geq 7$ standard drinks per week, or $\geq 2$ episodes of drinking of $\geq 4$ drinks on the same occasion. FASD with sentinel facial features (SFF) may be diagnosed in the absence of confirmed above-risk PAE given the specificity of simultaneous presentation of three SFFs to PAE.), measurement of SFF (There three features include: (1) palpebral fissure length $\geq 2$ SDs below the mean ( $<3$ rd percentile), (2) philtrum rated 4 or 5 on a five-point scale of the University of Washington (UW) Lip-Philtrum Guide and (3) upper lip rated 4 or 5 on a five-point scale of the UW Guide. ${ }^{26}$ ), assessment of neurodevelopmental functioning in 10 domains (The 10 neurodevelopmental domains, as outlined in the Canadian Diagnostic Guideline, include: motor skills; neuroanatomy/neurophysiology; cognition; language; academic achievement; memory; attention; executive function, including impulse control and hyperactivity; affect regulation; and adaptive behaviour, social skills or social communication.) and FASD diagnostic outcome. Associated features of FASD are also recorded, as well as comprehensive information about the client's physical and mental health and well-being, including comorbidities, medication, substance use and difficulties in daily living. Finally, data are collected on recommendations for intervention, and on whether these recommended services are available near the client's home (see online supplemental appendix 1 for full questionnaire and table 1 for data collected for this study).

As of June 2021, the database contained more than 3500 records collected between 2010 and 2021. All individuals were evaluated by a multidisciplinary team according to the Canadian Diagnostic Guidelines for FASD. ${ }^{7}$ Of the individual records that included a diagnostic outcome, $62 \%$ received an FASD diagnosis (53\% without SFF and $9 \%$ with SFF), and $11 \%$ were designated at risk of neurodevelopmental disorder (NDD)/FASD. The mean age of individuals was 14 years old (range 0-60 years), and 59\% of the sample identified as male.

\section{Patient and public involvement}

Anecdotally, patients, clinicians and families have reported that they want to learn about FASD and its presentation with respect to brain impairment and physical and mental health comorbidities and, most importantly, bring a critical perspective to the work. The goal of this enhanced understanding is to inform more targeted and effective supports and services. Individuals with FASD want to know if their experiences are similar to the experiences of others with the same diagnosis, so they can contribute to the advancement of research. ${ }^{27}$ Recognising the valuable perspectives of individuals with FASD and their family members, as well as the clinical expertise of FASD diagnosticians, these stakeholders played an integral role in the development and design of the national database. Data fields in the database and their indicators were developed by a rigorous process involving the input of diagnosticians and family members of those with FASD (the public), and adults with FASD (patients) across Canada and internationally. Feedback was sought from these stakeholders to ensure that data collection would be feasible and analysis would provide meaningful information and results.

\section{Process of stakeholder engagement}

In 2005, the Canada FASD Research Network (CanFASD) administered a survey to the designated departmental leads from the seven provincial/territorial ministries that supported the research to identify current and future priorities for FASD-related research, projects and programmes. One of the top identified priority areas was to build the capacity of multidisciplinary diagnostic clinics to work together to contribute evidence to the field of FASD diagnosis in Canada. In order to better understand the gaps and opportunities in this area, CanFASD hosted 
Table 1 Data collected

\section{Demographics}

Historical data

Diagnostic criteria

Diagnostic outcome

Associated features

Physical health comorbidities

Recommendations

Difficulties in daily living

Mental health comorbidities

\section{Age; gender; living situation; region}

Prenatal exposure to other substances; family history of FASD; trauma; attachment issues; physical or sexual abuse

Confirmation of PAE; facial measurements; neurodevelopmental functioning

FASD with SFF; FASD without SFF; at risk for neurodevelopmental disorder/FASD; no FASD

Sleep problems; sensory sensitivities; sensory processing issues; slow processing speed; gender identity issues

Congenital malformations; auditory deficit; visual deficit; growth restriction; failure to thrive; microcephaly; neurological conditions; head and neck issues; cleft lip/ palate; cardiovascular conditions; respiratory problems; endocrinological condition; musculoskeletal condition; infectious disease

Intellectual disability; attention deficit hyperactivity disorder; attachment disorder; developmental coordination disorder; language disorder/impairment; Tourette syndrome; anxiety disorder; mood disorder; autism spectrum disorder; bipolar disorder; conduct disorder; oppositional defiant disorder; obsessive compulsive disorder; post-traumatic stress disorder; schizophrenia; substance use disorder; suicidality

Coaching or support; FASD-specific (education or intervention); counselling (support group, individual therapy or couples/family); respite or daycare; substance use treatment; sexual health education; anger management; spousal abuse intervention; mental health support; basic needs (income support, food bank, safety precautions); guardianship, power of attorney, personal directive or other substitute decision making; child protection; legal services (legal aid, services for civil or family court issues); allied health services (speech and language pathologist, occupational therapy, behaviour therapy); medication/psychopharmacology or medical referral; accommodations/adaptation in environment, expectations, supports or routine; anticipatory guidance/prevention; reassessment

School problems (requiring teacher assistants, expulsion/suspension); employment problems; problems with living independently; housing problems (requiring assisted or sheltered housing); legal problems (victimisation, offending, custody/family court issues, incarceration)

FASD, fetal alcohol spectrum disorder; PAE, prenatal alcohol exposure; SFF, sentinel facial features.

a National Forum and invited representatives from every FASD diagnostic clinic in Canada, caregivers who represented families with FASD, as well as senior researchers in the field of FASD diagnosis at the time. One hundred and eighteen participants met over a 2-day period for facilitated discussions focused on the following questions:

- In what ways can cross-regional networking of FASD clinical information enhance or advance clinical research and knowledge transfer?

- What are the potential conflicts of interest and solutions that need to be considered?

- How should data be managed and controlled? What issues must be considered in data collection, data transfer, data storage, data access, data usage and data ownership?

- How can diagnostic clinics across Canada work together over the next 6 months to develop a process for a dataset that would be clinically relevant and helpful in knowledge transfer?

Forum participants identified a critical need for standardised data collection by FASD diagnostic clinics across Canada, based on simioar norms and using the same set of neuropsychological tests across clinics. They concluded that having all Canadian clinics contribute to a common dataset would provide an adequate sample size to develop Canadian norms for measures with existing norms derived from other countries (ie, growth charts). It was also anticipated that a common dataset would lead to a more accurate and helpful diagnostic system, including physical measures (dysmorphology), brain images and functional (psychometric) measures of the brain.

A working group was then developed to translate the recommendations of the National Forum into a process for data collection. Working group members were invited by CanFASD, based on experience and expertise in the field of FASD diagnosis. Members included paediatricians $(n=3)$, a clinical geneticist $(n=1)$, social workers $(n=2)$, FASD diagnostic clinic coordinators $(n=4)$, psychologists $(n=4)$, parents of individuals with FASD $(n=2)$, speech and language pathologists $(n=2)$ and FASD researchers $(n=2)$. The group had representation from eastern, western and central Canada and met in person for 4 days over the course of 1 year (2006). From these meetings, datafields were developed that were based on the diagnostic criteria 
of the 2005 Canadian Guidelines for Diagnosis ${ }^{28}$ currently in use at that time. Each datafield was discussed individually and combined into a form, which was streamlined as much as possible to reduce undue burden to data entry personnel. The ultimate goal of the form was to provide data that would:

- Be meaningful to FASD diagnostic clinics to help them better understand their population and to anticipate supports and services.

- Be meaningful to individuals with FASD and their families to better understand their disability and to receive effective recommendations.

- Contribute evidence to the FASD research field.

- Help policy-makers with information they need to advocate for and to implement policies, programmes and services related to FASD in their jurisdictions.

The data collection form was then piloted with two of Canada's largest diagnostic clinics who each used it for five patients. Feedback from the pilot was incorporated into the form, and in 2007-2008, the form was sent to every diagnostic clinic in Canada, along with a data dictionary and instructions. Clinics were contacted to gauge their interest and invited to an introductory teleconference with the working group. A template for patient consent and for ethics application was also provided. Over the next 4 years, clinics navigated the process of establishing datasets in their jurisdications with support from the working group and by 2012, 307 forms were submitted by four provinces.

With publication of the updated FASD Diagnostic Guidelines in Canada, ${ }^{7}$ it became necessary to update the datafields. The working group surveyed all clinics participating in data collection and received feedback about the process and utility of the data collected ( $n=48$ clinics responded). The working group also shared the form and sought feedback from experts in the USA $(n=4)$, Australia $(n=1)$ and New Zealand $(n=2)$ who also had FASD data collection systems. The working group met in person twice over the next year to refine the form as well as to identify an online platform for data entry and hosting. Two in-person workshops (2 hours each) were hosted with participation from families, individuals with FASD, clinicians, researchers and clinic coordinators who were attending FASD conferences and wished to attend $(n=68)$. The focus of discussion during these workshops was on the datafields and the process for data collection via the new online platform. Feedback was incorporated by the working group, and the online 'Dataform' was created in both English and French. An information package was then sent to each diagnostic clinic in Canada $(n=65)$ along with a clinic code for data entry and access to the online system.

A unique and important element of stakeholder engagement in this project $h$ the involvement of families (the public) and individuals with FASD (patients). These stakeholders participated extensively in developing the datafields that comprise the database, and helped to define the scope of the dataset, especially related to recommendations. For example, adults with FASD reported that they wanted to obtain more information on the trajectory of physical and mental health comorbidities across the lifespan, and their specific requests were included as indicators. Clinics and families who participated in the development of the database also helped to define the project's research questions and will continue to do so on an ongoing basis. Regular communication with clinics including conference calls, annual face-toface meetings, quarterly newsletters and individual clinic updates allows for ongoing collaboration, data quality assessment and refinement of the data collection process. To ensure that knowledge from the database is translated meaningfully, feedback and data are provided on a biannual basis to each participating clinic for their own use and comparison with provincial and national aggregate datasets. Results are disseminated in a format that clinics can share with their patients and families. Findings from the database have also been (and will continue to be) presented at various national and international meetings that are attended by individuals with FASD and their family members.

\section{Data analysis plan}

Statistical analyses will be performed biannually on datasets extracted in the fall (September 30) and spring (April 30) of each year, using SPSS Statistics V.27 software. All data will be grouped categorically. For demographic information, data will be coded as follows: age cohort (0-5 years, 6-12 years, 13-17 years, $18+$ years), gender (male, female, other), living situation (independent, with biological mother, biological father, other family member(s), foster care (non-family), adoptive parent(s), group home, homeless, in custody, other) and region (Northern and Western Canada, the Prairies, Central Canada, Atlantic Canada). For diagnostic criteria, confirmation of PAE will be coded as present, absent or unconfirmed/unknown; facial measurements will be coded as the number of SFF present $(0,1,2,3$ or inconclusive); neurodevelopmental functioning in each domain will be coded dichotomously (significantly impaired vs not significantly impaired); and diagnosis will be coded as one of four outcomes (FASD with SFF, FASD without SFF, at risk for NDD/FASD, no FASD). All other data will be coded dichotomously as either absent or present.

Descriptive statistics will be used to characterise the sample for categorical data. We will conduct Pearson $\chi^{2}$ tests and logistic regression to compare patterns between groups, examine predictive factors and explore strengths of association. Where available, prevalence data (eg, comorbidities) will be compared with rates found in neurotypical populations.

\section{Research question 1}

What is the neurodevelopmental profile of individuals assessed for FASD in Canada? How does it compare to profiles of individuals assessed for FASD in other countries? 
The neurodevelopmental profile of individuals assessed for FASD will be described in terms of the frequencies and patterns of neurodevelopmental impairment, and associated difficulties. Profiles and patterns of each diagnostic criterion (ie, confirmation of PAE, facial measurements, neurodevelopmental functioning) will be compared between diagnostic outcomes, age cohorts and genders. Findings in this area will provide valuable information about the profile of needs of individuals with FASD, and improve our understanding of where interventions may be targeted to improve outcomes for individuals with FASD. In addition, we will examine how the profile of neurodevelopmental needs in the Canadian population of individuals assessed for FASD compares to that in other countries. This will be possible through our established partnerships with FASD experts, researchers and clinicians in Australia, the UK and the USA, all of whom have been working to develop their own national FASD databases similar to that in Canada.

\section{Research question 2}

What are the physical and mental health comorbidities associated with FASD? How do these rates compare to the general population?

The frequencies and patterns of health comorbidities among individuals assessed for FASD will be examined, and compared across diagnostic outcomes, age cohorts and genders. The strengths of association will be examined between physical and mental health comorbidities and diagnostic outcomes, pattern of brain impairment and difficulties in daily living. This information will allow for a more holistic and comprehensive understanding of the needs of individuals with FASD across the lifespan and will uncover areas of difficulty that may warrant additional services and supports. To compare the rates of co-occurring physical and mental health conditions in FASD with those in the general population, we will use existing data published in the academic ${ }^{2930}$ and grey ${ }^{31}$ literature.

\section{Research question 3}

A. Which non-diagnostic factors are the most strongly predictive of FASD?

With this question, we aim to identify the combinations of demographic, historical, physical and mental health, and adversity factors that are most strongly associated with being diagnosed with FASD for different age cohorts and genders. We will also explore the strengths of association between predictive factors and FASD diagnosis (any FASD diagnosis and specific FASD diagnostic categories). Predictive models will be developed to determine sensitivity and specificity of combinations of factors associated with being diagnosed with FASD. It is anticipated that findings from these analyses will further refine FASD diagnostic criteria, and lead to more sensitive screening tools across the life span.

B. Which diagnostic and individual factors are the most strongly predictive of FASD?
Diagnostic criteria data will be analysed collectively, independently and interdependently to explore which criteria may always co-occur, which are exclusive and predictive of FASD, and how non-diagnostic factors including age, gender, history or comorbidities may influence whether an individual receives an FASD diagnosis.

\section{Research question 4}

What are the most common recommendations for interventions for individuals assessed for FASD?

The frequency and pattern of recommendations made for each diagnostic outcome, age cohort, gender and region will be examined. We will also explore whether and how different types of recommendations are associated with specific areas of brain impairment and other physical and mental health comorbidities. Recommendations will be compared across regions to develop intervention maps for understanding what services are needed, and where they may be lacking. This information will allow us to better understand practical areas where individuals with PAE require support across their lifespan, and what factors influence the recommendations made. This information will be useful for clinicians to influence policy and practice and advocate for consistency in service availability across the country.

\section{Research question 5}

What factors may contribute to or protect against the difficulties in daily living associated with FASD?

To explore this question, we will characterise and compare difficulties in daily living across diagnostic outcomes, age cohorts and genders. We will also examine the strengths of association between difficulties in daily living and demographic and historical factors, diagnostic criteria, comorbidities and associated features. Although data in the database are cross-sectional, this examination will allow us to identify factors that may be related to higher rates of difficulties in daily living across the life span, and circumstances within which supports may be introduced and optimised.

\section{ETHICS AND DISSEMINATION}

Ethics approval for this project was obtained from the Ottawa Health Science Network Research Ethics Board (protocol \# 20160423-0H1), and is renewed on an annual basis. The database is hosted on the secure REDCap platform at the University of Alberta, in Edmonton, Alberta, Canada. REDCap is an important tool for data access, linkages and mobilisation. On agreeing to participate in the project, clinics receive a random identification code, and the principal investigator and statistics team is blind to the coding.

Researchers who wish to use the data for their own work are required to obtain approval from their own institutional ethics boards, and apply to a database oversight committee. Applications must align with the intent and ethics of the overall project. On approval, an anonymised, 
aggregated dataset is downloaded from the server and sent to the researchers via a secure, password-protected link. This external use of data stimulates the development of new research questions and collaborations, and expands the potential impact of the database.

Several studies have been published from the database $^{52532}$ and many more are underway. As new knowledge is gained, findings will be disseminated through presentations at local, national or international meetings; publications in academic and grey literature; and regular feedback to participating clinics, all with the goal of informing FASD research, practice and policy.

\section{DISCUSSION}

The National FASD database provides rich information, both medical and behavioural, about individuals assessed for FASD in Canada across the lifespan. This information contributes evidence related to diagnostic criteria, determining the need for and availability of intervention supports and stimulating further research. Information collected in the database will improve our understanding of the challenges, clinical profiles, functional needs and outcomes of Canadians who are exposed to alcohol prenatally. We know that Canadians presenting at FASD clinics experience substantial difficulties navigating daily life, ${ }^{5}$ and continued data collection and analysis through the database has important implications for guiding practice and policy responses to improve quality of life for these individuals and their families. The database also captures important information about individuals who are assessed for FASD but are not diagnosed. Although evidence in this area is limited, researchers suggest that clinically referred individuals with PAE who do not meet the criteria for a formal diagnosis may nonetheless experience complex needs requiring timely care ${ }^{533}$ Information on the functional needs and complex presentations of all Canadians with PAE allows for a comprehensive understanding of areas where supports are needed, and guides efforts to provide the most appropriate services and interventions.

Collecting information from Canadians with PAE across the lifespan also allows us to understand more about the trajectory of FASD in Canada, whether the common experiences of Canadians with FASD change systematically over time, and how services and policies should be modified to meet these changing needs. The database allows us to compare the profiles and characteristics of Canadians with FASD to other subgroups of the population to identify unique or pressing needs. Examining trends in FASD data at a regional level will allow us to determine whether the needs of individuals with FASD differ in specific locations, and whether tailored approaches to service delivery are needed and available in different parts of the country. Similarly, findings from the database project will reveal important information about the gaps between FASD diagnosis and service availability for families impacted by FASD. Individuals with FASD and their caregivers require access to coordinated supports and services that are informed by the pattern of brain impairment identified during the diagnostic assessment. ${ }^{30}$ In the current service system, these supports may be lacking, and findings from the database will highlight the most common priorities for intervention, as well as the most significant gaps in FASD services.

Finally, the database provides a structure for active communication and collaboration among all clinics in Canada that provide FASD diagnostic services. Already, there is preliminary data to suggest that FASD clinicians are operating with a good deal of consistency across the country, ${ }^{34} 35$ which may in part be attributable to engagement with the national database. This coordinated approach allows for a consistent application of FASD best practices, a cohesive community of practice and a stronger network of experts working together to support improved outcomes for individuals with FASD and their families.

\section{Limitations and challenges}

The database project has several limitations. First, despite our goal to have every diagnostic clinic in Canada (approximately 60 to date) contributing to the database, some jurisdictions are not represented. We have made significant efforts to recruit clinics from every Canadian province and territory, and to reduce barriers to participation, we continue to assist clinics with their local ethics applications. Nonetheless, there are regional gaps in the data that limit nationwide conclusions. Second, because the information in the database is cross-sectional, it is not possible to examine longitudinal trends or to follow up with individuals to see how their profiles and needs change throughout their lifespan. However, because data are collected from individuals at various life stages, general snapshot observations can be made about different experiences or challenges that may be most relevant for individuals with FASD as they age. Relatedly, with this project, we will be able to identify important focal points that warrant follow-up using longitudinal approaches to best understand this population. In addition, since the database is a clinical dataset rather than a true research database, there is no control group of individuals who are neurotypical, or of individuals who have PAE but do not experience problems significant enough to trigger a referral for assessment. Therefore, in order to contextualise findings from the database, we typically must compare results with existing literature from neurotypical populations (eg, prevalence of mental health disorders). Importantly, although the database provides a mechanism for uncovering areas of relative strength or absence of deficit among individuals assessed for FASD, in future iterations of the database, we will consider more targeted approaches and methods for identifying strengths-based characteristics, skills and assets that may be leveraged to support positive outcomes in this population.

Additional limitations relate to the data collected on PAE. Currently, the database does not include 
information about amount or type of alcohol consumed, nor does it include the specific timing of exposure during pregnancy. Moreover, although 'confirmed absent' PAE refers to no alcohol exposure, and confirmed PAE indicates exposure 'at or above risk levels' as specified in the Canadian Diagnostic Guideline, ${ }^{7}$ exposure levels between 'none' and 'above risk' are not captured. Most (if not all) clinics only accept individuals for an assessment if they meet or exceed the minimum PAE threshold.

The legal, ethical and administrative processing that is necessary to conduct research of this scope across jurisdictional lines is possible, but arduous, and may limit the level of detail included in the Database. A great deal of consideration was given to the development of each question, balancing the need to derive meaningful information with the priority that data entry must not be burdensome for clinics. However, through clinic consultation, we have learnt that additional valuable information would be available for collection in future iterations of the database. For instance, although in-depth information regarding the amount and timing of PAE was thought to be unattainable at the time of the Database development, we have learnt that most clinics have access to this information and that it would be feasible to collect in the future.

Finally, although the database is structured according to the Canadian FASD Diagnostic Guidelines, ${ }^{7}$ and guidance is provided to clinics for measuring and reporting on the diagnostic criteria, including a data dictionary, information in the dataset still comes through various avenues. These include self-report, record review or screening tools, and this variability may result in inconsistent reporting. In order to mitigate this, participating clinics have been provided with a list of recommended assessment tools for each of the measurements (as per the current Canadian diagnostic guideline), where appropriate. Clinics also use a collaborative online platform to share ideas and experiences related to data field interpretation and data entry, in order to increase consistency in the use of the database. Without funding for each clinic, it is necessary to rely on the enthusiasm and investment of clinicians to sustain the partnership. Without the efforts of the participating clinics and the individuals and families who consent to their data collection, the database would not be possible.

\section{CONCLUSION}

Canada's National FASD database provides an important framework for characterising and exploring the needs and outcomes of individuals with PAE across the life span. The comprehensive and nation-wide scope of the database enables researchers to examine questions that have not previously been possible to explore. The database provides a unique and timely opportunity to monitor the prevalence of FASD and associated health comorbidities at a population level, as well as evidence to determine optimal interventions mapped to physical, mental and neurodevelopmental issues and optimise developmental trajectories of individuals prenatally exposed to alcohol. The clinical presentation of Canadians with PAE and FASD is highly complex, and information derived from the database provides direct evidence of areas where supports are needed. Critically, this information can guide our efforts to provide the most appropriate services and interventions to support positive outcomes for individuals with FASD, their caregivers, families and communities.

\section{Twitter Jocelynn Cook @JocelynnCook}

Acknowledgements We would like to thank the many clinicians, individuals with FASD and their families from across Canada who provided input into the development of this project, who helped us to develop and to refine the data collection fields and who provided advice on how to shape the research questions. We would also like to acknowledge all participating diagnostic clinics, the individuals with FASD and the families that consent to contributing their information.

Contributors $\mathrm{JC}$ led the conceptualisation of the design of this project, the applications for funding and the overall development of the database. KU led the recruitment of participants and clinics, development of the knowledge translation plan and the reporting of the work. KF refined research questions, piloted the survey tool and provided interpretation of the data. All authors drafted sections of the manuscript and revised it critically. All approve this final version for publication and agree to be accountable for all aspects of the work.

Funding This work was participally supported by the Public Health Agency of Canada (Contribution Agreement \#1617-HQ-000052) and Kids Brain Health Network (NCE Grant \#20000).

Disclaimer Neither funder had any involvement in in the study design; in the collection, analysis and interpretation of the data; in the writing of the report, or in the decision to submit the paper for publication.

Competing interests None declared.

Patient consent for publication Not required.

Provenance and peer review Not commissioned; externally peer reviewed.

Supplemental material This content has been supplied by the author(s). It has not been vetted by BMJ Publishing Group Limited (BMJ) and may not have been peer-reviewed. Any opinions or recommendations discussed are solely those of the author(s) and are not endorsed by BMJ. BMJ disclaims all liability and responsibility arising from any reliance placed on the content. Where the content includes any translated material, BMJ does not warrant the accuracy and reliability of the translations (including but not limited to local regulations, clinical guidelines, terminology, drug names and drug dosages), and is not responsible for any error and/or omissions arising from translation and adaptation or otherwise.

Open access This is an open access article distributed in accordance with the Creative Commons Attribution Non Commercial (CC BY-NC 4.0) license, which permits others to distribute, remix, adapt, build upon this work non-commercially, and license their derivative works on different terms, provided the original work is properly cited, appropriate credit is given, any changes made indicated, and the use is non-commercial. See: http://creativecommons.org/licenses/by-nc/4.0/.

ORCID iDs

Jocelynn Cook http://orcid.org/0000-0002-1936-0669

Katherine Flannigan http://orcid.org/0000-0001-7230-2532

\section{REFERENCES}

1 Maggi S, Irwin LJ, Siddiqi A, et al. The social determinants of early child development: an overview. J Paediatr Child Health 2010;46:627-35.

2 Guhn M, Zumbo BD, Janus M, et al. Validation theory and research for a population-level measure of children's development, wellbeing, and school readiness. Soc Indic Res 2011;103:183-91.

3 Janus M, Brownell M, Reid-Westoby C, et al. Establishing a protocol for building a pan-Canadian population-based monitoring system for early childhood development for children with health disorders: 
Canadian children's health in context study (CCHICS). BMJ Open 2018;8:e023688.

4 Connor PD, Streissguth AP. Effects of prenatal exposure to alcohol across the life span. Alcohol Health Res World 1996;20:170-4.

5 McLachlan K, Flannigan K, Temple V, et al. Difficulties in daily living experienced by adolescents, transition-aged youth, and adults with fetal alcohol spectrum disorder. Alcohol Clin Exp Res 2020;44:1609-24.

6 Streissguth A, Barr H, Kogan J. Understanding the occurrence of secondary disabilities in clients with fetal alcohol syndrome (Fas) and fetal alcohol effects (Fae): final report to the centers for disease control and prevention. Seattle, Washington: Centers For Disease Control And Prevention, 1996: 1-72.

7 Cook JL, Green CR, Lilley CM, et al. Fetal alcohol spectrum disorder: a guideline for diagnosis across the lifespan. CMAJ 2016;188:191-7.

8 Burgess DJ, Moritz KM. Prenatal alcohol exposure and developmental programming of mental illness. $J$ Dev Orig Health Dis 2020;11:211-21.

9 Easey KE, Dyer ML, Timpson NJ, et al. Prenatal alcohol exposure and offspring mental health: a systematic review. Drug Alcohol Depend 2019;197:344-53.

10 Mattson SN, Bernes GA, Doyle LR. Fetal alcohol spectrum disorders: a review of the neurobehavioral deficits associated with prenatal alcohol exposure. Alcohol Clin Exp Res 2019;43:1046-62.

11 Price A, Cook PA, Norgate S, et al. Prenatal alcohol exposure and traumatic childhood experiences: a systematic review. Neurosci Biobehav Rev 2017;80:89-98.

12 Streissguth AP, Bookstein FL, Barr HM, et al. Risk factors for adverse life outcomes in fetal alcohol syndrome and fetal alcohol effects. $J$ Dev Behav Pediatr 2004;25:228-38.

13 Koponen AM, Kalland M, Autti-Rämö I. Caregiving environment and socio-emotional development of foster-placed FASD-children. Child Youth Serv Rev 2009;31:1049-56.

14 McLachlan K, Andrew G, Pei J. Assessing FASD in young children: exploring clinical complexities and diagnostic challenges. J Popul Ther Clin Pharmacol 2015;22:E108-24.

15 Fagerlund A, Autti-Rämö I, Hoyme HE, et al. Risk factors for behavioural problems in foetal alcohol spectrum disorders. Acta Paediatr 2011;100:1481-8.

16 Koponen AM, Kalland M, Autti-Rämö I, et al. Socio-emotional development of children with foetal alcohol spectrum disorders in long-term foster family care: a qualitative study. Nordic Social Work Research 2013;3:38-58.

17 Quan R, Brintnell ES, Leung AWS. Elements for developing community-based interventions for adults with fetal alcohol spectrum disorder: a scoping review. Br J Occup Ther 2019;82:201-12.

18 Culshaw M. Individuals with fetal alcohol spectrum disorder: predictive factors for successful occupational performance. ProQuest Dissertations Publishing, 2015.

19 Duquette C, Stodel E. School experiences of students with fetal alcohol spectrum disorder. Exceptionality Education Canada 2005; 15:51-75.
20 Currie BA, Hoy J, Legge L, et al. Adults with fetal alcohol spectrum disorder: factors associated with positive outcomes and contact with the criminal justice system. J Popul Ther Clin Pharmacol 2016;23:E37-52.

21 Popova S, Lange S, Chudley AE. World Health organization International study on the prevalence of fetal alcohol spectrum disorder (FASD). Canadian Component, Centre for Addiction and Mental Health, 2018

22 Thanh NX, Jonsson E, Salmon A, et al. Incidence and prevalence of fetal alcohol spectrum disorder by sex and age group in Alberta, Canada. J Popul Ther Clin Pharmacol 2014;21:e395-404.

23 Kodituwakku PW. Neurocognitive profile in children with fetal alcohol spectrum disorders. Dev Disabil Res Rev 2009;15:218-24.

24 Mattson SN, Crocker N, Nguyen TT. Fetal alcohol spectrum disorders: neuropsychological and behavioral features. Neuropsychol Rev 2011;21:81-101.

25 Clarren S, Halliwell Cl, Werk CM, et al. Using a common form for consistent collection and reporting of fasd data from across Canada: a feasibility study. J Popul Ther Clin Pharmacol 2015;22:e211-28.

26 Astley SJ, Clarren SK. Measuring the facial phenotype of individuals with prenatal alcohol exposure: correlations with brain dysfunction. Alcohol Alcohol 2001;36:147-59.

27 Himmelreich M, Lutke C, Hargrove E. The lay of the land: Fetal Alcohol Spectrum Disorder (FASD) as a whole body diagnosis. In: Begun AL, Murray MM, eds. The Routledge Handbook of social work and addictive behaviors. 1st edn. Taylor and Francis, 2020.

28 Chudley AE, Conry J, Cook JL. Public Health Agency of Canada's National Advisory Committee on Fetal Alcohol Spectrum Disorder. Fetal alcohol spectrum disorder: Canadian guidelines for diagnosis. CMAJ 2005;172:S1-21.

29 McRae L, O'Donnell S, Loukine L, et al. Report summary - Mood and Anxiety Disorders in Canada, 2016. Health Promot Chronic Dis Prev Can 2016;36:314-5.

30 Bernes GA, Villodas M, Coles CD, et al. Validity and reliability of executive function measures in children with heavy prenatal alcohol exposure: correspondence between multiple Raters and laboratory measures. Alcohol Clin Exp Res 2021;45:596-607.

31 Statistics Canada. Table 13-10-0096-11 heavy drinking, by age group, 2020.

32 Temple VK, Cook JL, Unsworth K, et al. Mental health and affect regulation impairment in fetal alcohol spectrum disorder (FASD): results from the Canadian national FASD database. Alcohol Alcohol 2019;54:545-50.

33 Flannigan K, Gill K, Pei J, et al. Deferred diagnosis in children assessed for fetal alcohol spectrum disorder. Appl Neuropsychol Child 2019;8:213-22.

34 Coons-Harding KD, Flannigan K, Burns C, et al. Assessing for fetal alcohol spectrum disorder: a survey of assessment measures used in Alberta Canada. J Popul Ther Clin Pharmacol 2019;26:e39-55.

35 Flannigan KR, Coons-Harding KD, Turner O, et al. A survey of measures used to assess brain function at FASD clinics in Canada. Can Psychol 2020. doi:10.1037/cap0000245 